\title{
Spontaneous cerebrospinal fluid rhinorrhoea: computed tomography and magnetic resonance imaging findings
}

Chih Ching $\underline{C h o o n g}^{1}, \mathrm{MBChB}, \mathrm{FRCR}$, Sudhakar Kundapur Venkatesh ${ }^{2}, \mathrm{MD}, \mathrm{FRCR}$, Rajendra Vishnu $\underline{\text { Phadke }}^{3}$, MBBS, MD

ABSTRACT Spontaneous cerebrospinal fluid rhinorrhoea is a rare clinical entity. The accurate localisation of the leakage site is essential for surgical planning. Imaging techniques such as high-resolution computed tomography (CT), CT cisternography and magnetic resonance cisternography in variable combinations are performed for this purpose. This pictorial essay aims to present the spectrum of imaging findings in cases of spontaneous cerebrospinal fluid rhinorrhoea, which may be useful for radiologists in the determination of the site of cerebrospinal fluid leakage.

Keywords: imaging spontaneous cerebrospinal fluid leak

\section{INTRODUCTION}

Cerebrospinal fluid (CSF) rhinorrhoea occurs when there is communication between the intracranial subarachnoid space and nasal cavity or paranasal sinuses through osteodural defects at the base of the skull. CSF leaks can be classified according to aetiology, either as traumatic (accidental and iatrogenic) or nontraumatic. Nontraumatic CSF fistulas are further divided into high-pressure (associated with tumours or hydrocephalus) and normal-pressure leaks (associated with congenital anomalies). ${ }^{(1)}$ As spontaneous CSF fistula is idiopathic and accounts for only $3 \%-4 \%$ of leaks, ${ }^{(2)}$ it is considered a rare and separate entity from other nontraumatic causes.

A proposed theory of the cause of spontaneous CSF leaks is chronic pulsatile CSF forces exuding continual erosive action at the site of arachnoid granulations, forming small pits and defects in the inner table of the skull or sinus wall, eventually leading to osteodural fistulas and herniation of the brain tissue. ${ }^{(3)}$ Regardless of the aetiology, localising the site of CSF leak is of utmost importance, as it would enable appropriate management of the leak to prevent further rhinorrhoea and complications, such as low-pressure headache, intracranial abscess and meningitis. Imaging plays an important role, not only to determine the site and size of the skull base defect, but also to exclude secondary causes (such as tumours and congenital lesions) and detect associated meningoencephaloceles. For defects in the cribriform plate, ethmoid roof or central sphenoid sinus, the endoscopic transnasal approach is used, while defects in the lateral recess of the sphenoid sinus may be repaired using the endoscopic transpterygoid approach. An external approach using osteoplastic flaps is used for frontal sinus leaks. ${ }^{(4)}$

\section{CLINICAL FEATURES AND IMAGING}

Spontaneous CSF rhinorrhoea is most commonly found in obese, middle-aged women. ${ }^{(1,4)}$ The condition is usually insidious in its onset, with profuse intermittent nasal CSF discharge, exacerbated by Valsalva manoeuvres. ${ }^{(1)}$ Patients commonly present with headaches that may persist for months and even years. More importantly, there is a $4 \%$ risk of recurrent meningitis reported in cases of persistent CSF leak. A diagnosis of CSF rhinorrhoea can be confirmed by the presence of beta- 2 transferrin, a protein highly specific for human CSF, in nasal secretions. ${ }^{(1)}$

As nasal endoscopic surgery is increasingly performed for CSF rhinorrhoea, the use of imaging is important for surgical planning. Imaging may demonstrate the exact site of CSF leak and forewarn the presence of associated findings, such as a dehiscent optic nerve or internal carotid artery, and the presence of brain tissue herniation. Although no 'gold standard' imaging is available, non-contrast high-resolution computed tomography (HRCT), CT cisternography (CTC) and magnetic resonance cisternography (MRC) are the main imaging techniques utilised in the investigation of clinically suspected spontaneous CSF rhinorrhoea.

\section{HIGH-RESOLUTION CT}

With the introduction of multidetector CT scanners, rapid continuous volumetric images can now be obtained in the axial plane, allowing for coronal and sagittal multiplanar reformats using the raw data without compromising image resolution. ${ }^{(1)}$ Axial images are necessary in order to evaluate the posterior wall of the frontal sinus, the posterior and lateral walls of the sphenoid sinus, and the mastoid complex; whereas coronal images are best used to assess the cribriform plates, roof of the ethmoid and sphenoid

${ }^{1}$ Department of Diagnostic Radiology, National University Hospital, Singapore, ${ }^{2}$ Radiology, Mayo Clinic, Rochester, USA, ${ }^{3}$ Department of Radiodiagnosis, Sanjay Gandhi Post Graduate Institute of Medical Sciences, Lucknow, India

Correspondence: Dr Choong Chih Ching, Registrar, Department of Diagnostic Radiology, National University Hospital, 5 Lower Kent Ridge Road, Singapore 119074. chihchingc@gmail.com 



Fig. 1 A 28-year-old man presented with intermittent cerebrospinal fluid (CSF) rhinorrhoea related to postural changes. (a) Plain coronal CT image shows a bony defect (arrow) in the left cribriform plate, measuring $6 \mathrm{~mm} \times 5 \mathrm{~mm}$ with flattening of crista galli (curved arrow) on the same side. An adjacent contiguous soft tissue mass $\left(^{*}\right)$ extended into the left nasal cavity, causing a deviated nasal septum. (b) Coronal CT cisternography image clearly shows CSF leakage through the same defect with contrast filling of a large pouch (arrow) in the nasal cavity. No herniation of the brain tissue is noted. (c) Axial CT cisternography image shows a partially empty sella (arrow). At surgery, a well-defined, smooth defect was seen in the left cribriform plate and an arachnoid pouch was seen to herniate through the defect. The pouch was excised and the arachnoid membrane sutured, and finally, a dural graft was placed over the defect.
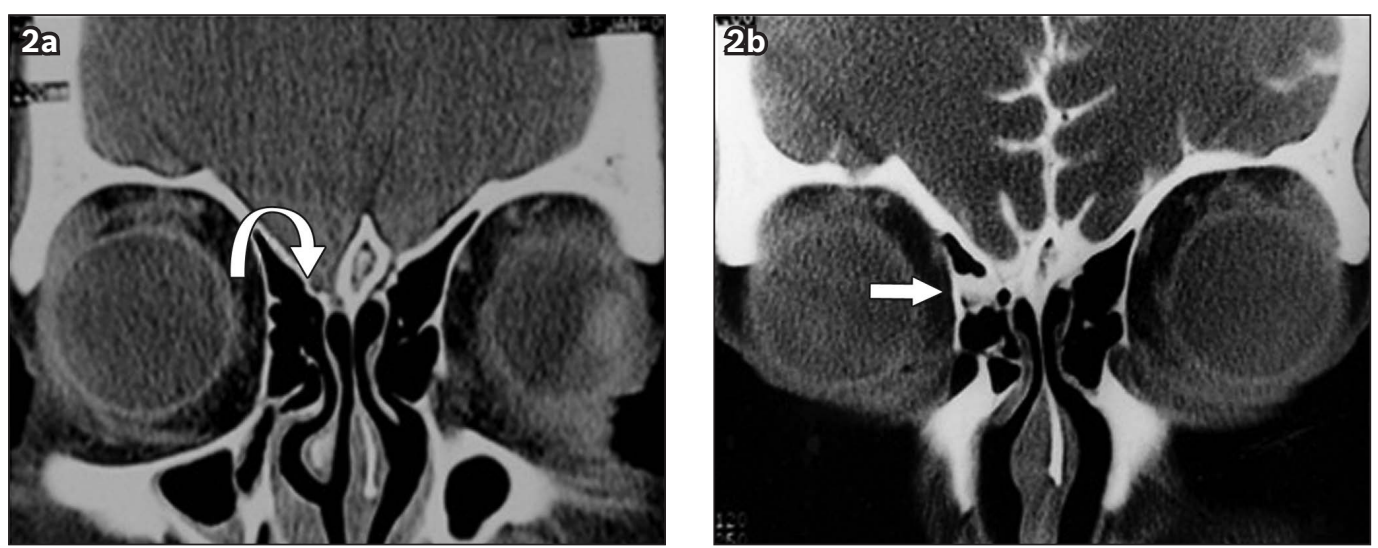

Fig. 2 A 36-year-old man with a one-year history of sporadic episodes of nasal discharge provoked by Valsalva manouevres presented for further investigation. (a) Plain coronal HRCT image shows a small osseous defect in the right cribriform plate (curved arrow); the adjacent paranasal sinuses were clear. (b) Coronal CT cisternography image performed with Valsalva manoeuvres clearly shows leakage of contrast into the adjacent ethmoid air cells (arrow), in keeping with an osteodural fistula.

sinuses. ${ }^{(1)}$ Plain HRCT using a multidetector scanner has a reported $92 \%$ sensitivity and $100 \%$ specificity in detecting bony defects in the skull base. ${ }^{(5)}$ It gives indirect evidence of the site of the CSF fistula and is not limited to the presence of an active CSF leak.

Positive CT findings in spontaneous CSF rhinorrhoea are characterised by the presence of a skull base defect with opacification or mucosal thickening of the contiguous sinus or air cells (Figs. 1a \& 2a). Although CSF leak is not directly visible on non-contrast HRCT, some studies have reported high sensitivities of $71 \%-93 \%$, with diagnostic accuracies that are superior to CT and MRC. ${ }^{(5-7)}$

\section{CT CISTERNOGRAPHY}

CTC involves CT imaging before and after intrathecal injection of iodinated contrast to opacify the CSF cisterns. In our centre, an intrathecal injection of $10 \mathrm{~mL}$ of $300 \mathrm{mg}$ iodine/mL, non-ionic contrast medium via lumbar puncture is typically performed, following which the patient is placed in the Trendelenburg position for 5-10 minutes. Patients are imaged in a prone position with the neck extended. Contiguous 3-5 mm coronal CT images are obtained. The images are processed in bone algorithm and viewed under wide windows and different level settings. CTC identifies active CSF leaks by demonstrating accumulation of contrast material in the nasal cavity or sinus (Figs. 1b, 1c \& 2b) adjacent to the site of the bony defect. An increase in Hounsfield units in the sinus fluid by $50 \%$ or more, as compared to the plain scan, is considered positive for contrast leakage. ${ }^{(7)}$

When related to the ethmoid sinus, the most common site of the fistula is along the cribriform plate or the course of the anterior ethmoid artery. ${ }^{(3,8)}$ Midline transsphenoidal fistulas are usually situated in the anteroinferior wall of the sella, which is both a zone of least resistance and an area most affected by the 

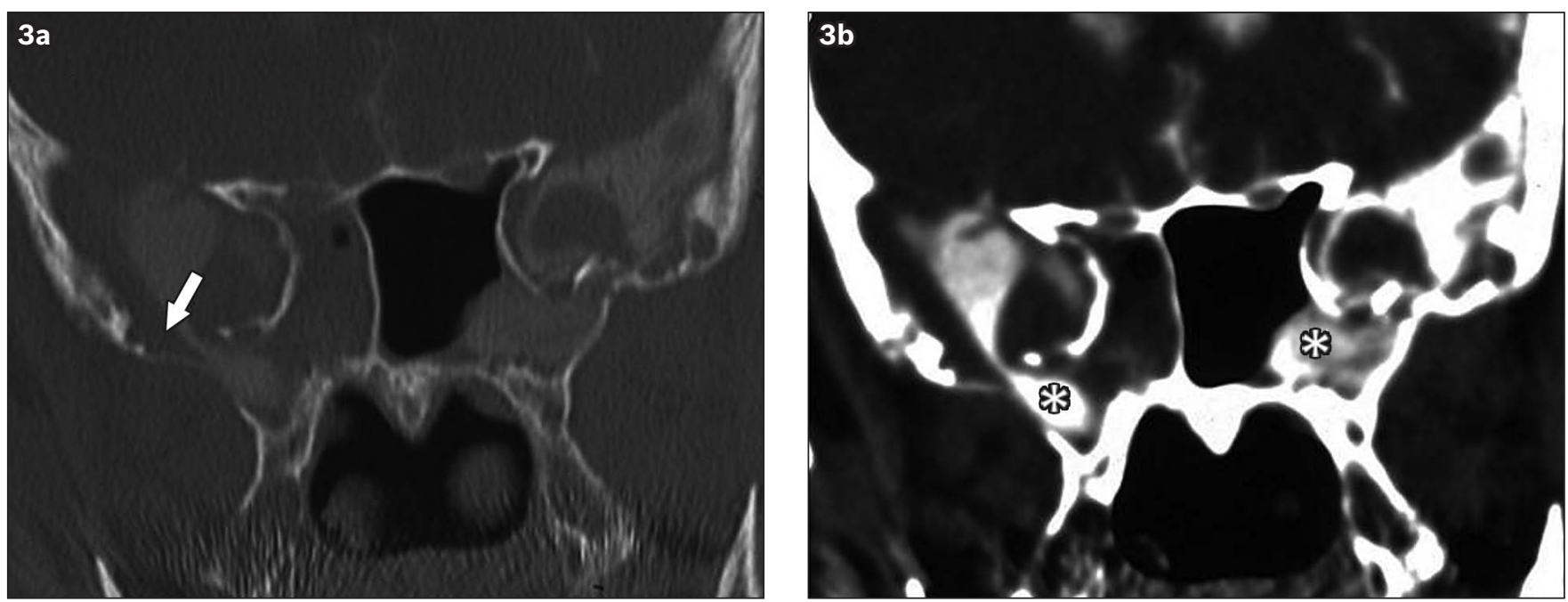

Fig. 3 A 54-year-old man was referred by his general practitioner for workup of chronic sinonasal disease, which subsequently led to CT cisternography. (a) On coronal bone algorithm, there were arachnoid granulation pits (arrow) and erosions in the skull base on both sides, with opacification of the adjacent lateral recesses of the sphenoid sinuses. (b) Coronal soft tissue window shows large meningocoeles (*) containing contrast herniating via the junction of the lateral wall of the sphenoid sinuses and the middle cranial fossae.
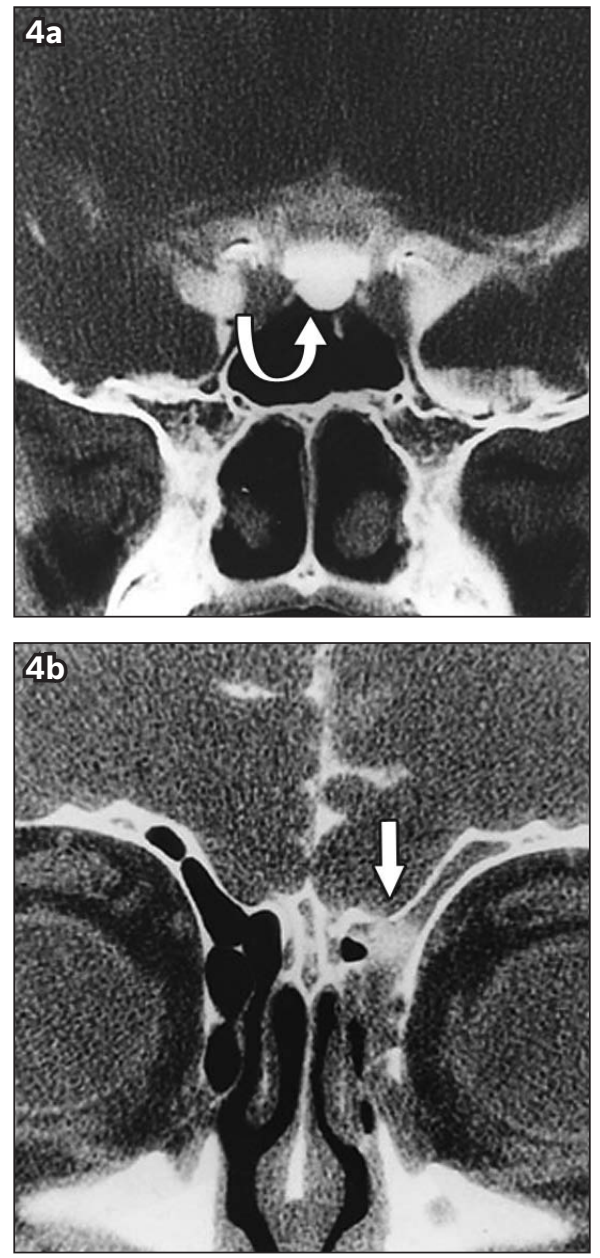

Fig. 4 A 45-year-old woman with a four-month duration of cerebrospinal fluid rhinorrhoea was investigated with CT cisternography. Coronal images post-intrathecal contrast administration show (a) an enlarged empty sella (curved arrow) completely opacified by contrast, with marked thinning of the sella floor; and (b) the site of contrast leak, identified anteriorly in the left frontal sinus (arrow) due to a defect in the fovea ethmoidalis.

pulsatile action of arachnoid diverticulum. ${ }^{(3)}$ When spontaneous CSF leak occurs at the sphenoid sinus, the associated findings on $\mathrm{CT}$ include extensive pneumatisation of the lateral recesses,

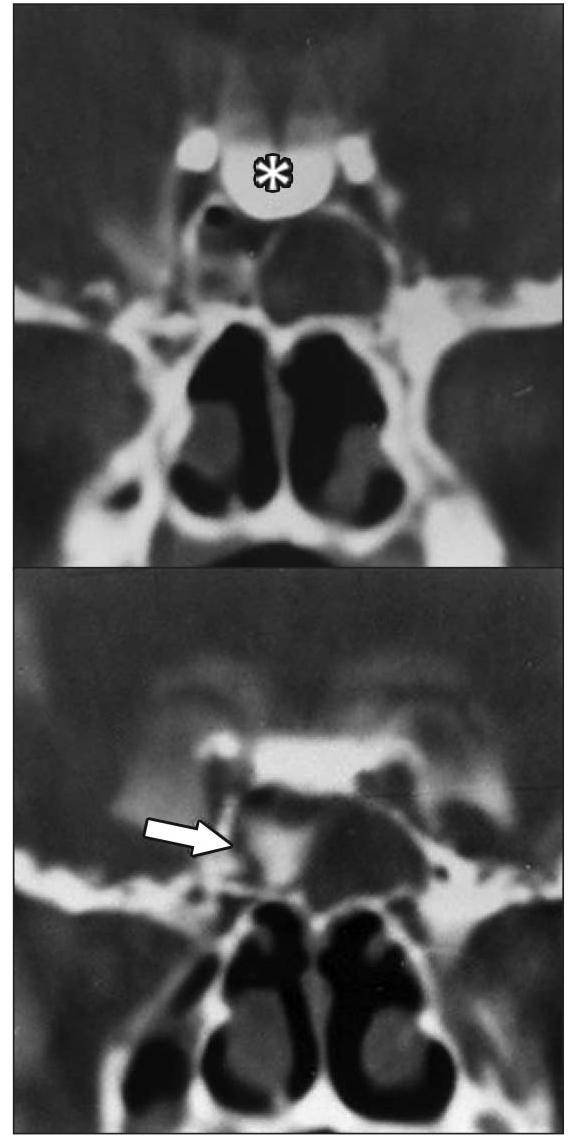

Fig. 5 Coronal CT cisternography images of a middle-aged man show an enlarged pituitary sella completely occupied by an opacified cerebrospinal fluid pouch $\left(^{*}\right)$, with cerebrospinal fluid leak from the sella floor demonstrated by contrast extravasation into the right sphenoid sinus (arrow).

outward concave orientation of the inferior portion of the lateral wall of the sphenoid sinus, arachnoid pits (Fig. 3) and an empty sella. ${ }^{(9)}$ An empty or partially empty sella is commonly associated with spontaneous CSF fistula (Fig. 4). ${ }^{(4,8)}$ When spontaneous CSF rhinorrhoea is associated with an empty sella and sella enlargement, the sella floor is the most likely site of leak (Fig. 5). ${ }^{(10)}$ 



Fig. 6 A 56-year-old woman who had meningitis and cerebrospinal fluid (CSF) rhinorrhoea. (a) Coronal plain CT image show defects on both sides of the cribriform plate (arrow) with mucosal thickening in the adjacent nasoethmoids. (b) CT cisternography image shows contrast leakage (arrowheads) from both osteodural defects. (c) MR cisternography image clearly shows two contiguous CSF columns (arrowheads) communicating with the adjacent sinonasal spaces.


Fig. 7 A 20-year-old student with spontaneous cerebrospinal fluid (CSF) rhinorrhoea complicated by two episodes of pneumococcal meningitis subsequently underwent endoscopic repair of the CSF leak due to the cribriform plate defect. (a) Plain coronal HRCT image shows a depression at the left of the cribriform plate and roof of the left anterior ethmoid cells $\left({ }^{*}\right)$, with opacification of the left ethmoid sinus. (b) Coronal CT cisternography image shows contrast leakage into the ethmoid sinus (arrow). (c) Coronal MR cisternography image shows depressed left cribriform plate and herniation of the left frontal cerebral tissue and meninges into the left ethmoid sinus (curved arrow), in keeping with a meningoencephalocele.

CTC allows for precise localisation of the bony defect, and is particularly useful in cases of multiple osseous defects with adjacent sinus opacification. ${ }^{(6)}$ However, the diagnostic value of CTC is limited by the absence of an active CSF leak at the time of imaging. Previous studies have reported low sensitivities of $33 \%$ and $40 \%{ }^{(7,11)}$ in the absence of an active CSF leak. Other limitations include a small defect size in which low-flow or small quantities of contrast leakage may not be detectable, and technical factors such as poor contrast opacification of the basilar cisterns. ${ }^{(6)}$ Lumbar puncture for intrathecal contrast injection is also relatively contraindicated in patients with active meningitis or raised intracranial pressure.

\section{MR CISTERNOGRAPHY}

Herniation of intracranial structures through the skull base defect in spontaneous CSF rhinorrhoea is better evaluated with MRC due to its superior soft tissue resolution. The knowledge of herniation contents before endoscopic surgical grafting procedure is essential, as bone and cartilage grafts are used only in the presence of herniation of the brain or meninges. ${ }^{(12)}$ MRC has a reported sensitivity of up to $92 \%$ and specificity of $100 \%{ }^{(5)}$ Although MR imaging is limited by the lack of osseous details and its inability to detect small bony defects, it is noninvasive and can demonstrate
CSF leak without the disadvantages of ionising radiation or lumbar puncture. Therefore, MRC complements HRCT in evaluating the contents of herniation when $\mathrm{CT}$ reveals the presence of an osteodural defect.

MRC is performed using standard head coils, heavily utilising T2-weighted, two- or three-dimensional techniques with fat suppression and multiple planes. This technique does not require intrathecal contrast injection or active CSF leakage. Preand post-gadolinium thin-section images help to differentiate mucosal thickening from meningocoele. ${ }^{(1)}$ Positive findings in MRC include a contiguous, high T2-weighted signal CSF column communicating between the subarachnoid space and the sinus or nasal cavity via the skull base (Fig. 6), or herniation of brain parenchyma and/or meninges extracranially (Fig. 7). There may be adjacent low-lying gyrus rectus, encephalomalacia secondary to traction and dural enhancement. ${ }^{(1)}$ However, false-positive findings may result from viscous secretions, inflammatory changes from sinusitis and susceptibility artefacts from bone-air interphase. ${ }^{(13)}$

The use of contrast-enhanced MRC derived from fat-saturated T1-weighted imaging post-intrathecal gadolinium injection has been shown to improve accuracy, especially with slow-flow CSF leaks. ${ }^{(14)}$ Intrathecal injection of gadolinium demonstrates 
excellent differentiation between CSF-containing spaces and adjacent bone, as well as soft tissue and paranasal sinuses, resulting in high diagnostic sensitivity and low false-negative results. ${ }^{(15)}$ Although several preliminary studies have shown that intrathecal gadolinium can be administered without major complications, it has not yet received FDA approval for this purpose. ${ }^{(13,15)}$

\section{CONCLUSION}

In this era of transnasal endoscopic repair of skull base defect in CSF rhinorrhoea, imaging is of paramount importance in the preoperative evaluation and precise localisation of the osteodural defect. The awareness of the individual roles of plain HRCT, CTC and MRC, as well as accurate characterisation of the fistula with its associated findings in cases of spontaneous CSF rhinorrhoea, will not only facilitate surgical planning, but can also increase the chance of successful dural repair, thereby lessening the possibility of recurrent explorations.

\section{REFERENCES}

1. Lloyd KM, DelGaudio JM, Hudgins PA. Imaging of skull base cerebrospinal fluid leaks in adults. Radiology 2008; 248:725-36.

2. Loew F, Pertuiset B, Chaumier EE, Jacshe H. Traumatic, spontaneous and postoperative CSF rhinorrhoea. Adv Tech Stand Neurosurg 1984; 11:169-207.

3. Ommaya AK. Cerebrospinal fluid rhinorrhoea. In: Eilkins RH, Rengachary SS, eds. Neurosurgery. New York: McGraw Hill Inc,1985: 1637-47.

4. Schlosser RJ, Bolger WE. Spontaneous nasal cerebrospinal fluid leaks and empty sella syndrome: a clinical association. Am J Rhinol 2003; 17:91-6.
5. Shetty PG, Shroff MM, Sahani DV, Kirtane MV. Evaluation of highresolution $C T$ and $M R$ cisternography in the diagnosis of cerebrospinal fluid fistula. AJNR Am J Neuroradiol 1998; 19:633-9.

6. Stone JA, Castillo M, Neelon B, Mukherji SK. Evaluation of CSF leaks: highresolution $\mathrm{CT}$ compared with contrast-enhanced $\mathrm{CT}$ and radionuclide cisternography. AJNR Am J Neuroradiol 1999; 20:706-12.

7. La Fata V, McLean N, Wise SK, DelGaudio JM, Hudgins PA. CSF leaks: correlation of high-resolution CT and multiplanar reformations with intraoperative endoscopic findings. AJNR Am J Neuroradiol 2008; 29:536-41.

8. Schuknecht B, Simmen D, Briner HR, Holzmann D. Nontraumatic skull base defects with spontaneous CSF rhinorrhea and arachnoid herniation: imaging findings and correlation with endoscopic sinus surgery in 27 patients. AJNR Am J Neuroradiol 2008; 29:542-9.

9. Shetty PG, Shroff MM, Fatterpekar GM, Sahani DV, Kirtane MV. A retrospective analysis of spontaneous sphenoid sinus fistula: MR and CT findings. AJNR Am J Neuroradiol 2000; 21:337-42.

10. García-Uría J, Carrillo R, Serrano P, Bravo G. Empty sella and rhinorrhoea. A report of eight treated cases. J Neurosurg 1979; 50:466-71.

11. Eljamel MS, Pidgeon CN, Toland J, Phillips JB, O'Dwyer AA. MRI cisternography, and the localization of CSF fistulae. Br J Neurosurg 1994; 8:433-7.

12. Carrau RL, Snyderman CH, Kassam AB. The management of cerebrospinal fluid leaks in patients at risk for high-pressure hydrocephalus. Laryngoscope 2005; 115:205-12.

13. Algin O, Turkbey B. Intrathecal gadolinium-enhanced MR cisternography: a comprehensive review. AJNR Am J Neuroradiol 2013; 34:14-22. http://doi: 10.3174/ajnr.A2899. Epub 2012 Jan 19.

14. Aydin K, Guven K, Sencer S, Jinkins JR, Minareci O. MRI cisternography with gadolinium-containing contrast medium: its role, advantages and limitations in the investigation of rhinorrhoea. Neuroradiology 2004; 46:75-80.

15. Arbeláez A, Medina E, Rodríguez M, Londoño AC, Castillo M. Intrathecal administration of gadopentate dimeglumine for MR cisternography of nasoethmoidal CSF fistula. AJR Am J Roentgenol 2007; 188:W560-4. 


\section{SINGAPORE MEDICAL COUNCIL CATEGORY 3B CME PROGRAMME} (Code SMJ 201303B)

Question 1. Regarding cerebrospinal fluid (CSF) rhinorrhoea:

(a) CSF rhinorrhoea is due to an osteodural defect in the skull base, which communicates with the nasal cavity or paranasal sinus.

(b) The most common cause of CSF rhinorrhoea is trauma.

(c) CSF rhinorrhoea in young, obese women is most commonly of the spontaneous type.

(d) Meningitis is a complication in about $20 \%$ of patients with persistent CSF leakage.

Question 2. Regarding high-resolution computed tomograpy (HRCT) and CT cisternography (CTC) in CSF rhinorrhoea:

(a) HRCT is the modality of choice in depicting the presence of bone erosion or defect in the base of the skull .

(b) The sensitivity of plain HRCT is dependent on the presence of an active CSF leak.

(c) When there are fluid levels, mucosal thickening or opacification of the sinus adjacent to the site of osseous defect, an active CSF leak or meningoencephalocele cannot be excluded.

(d) Following intrathecal injection of iodinated contrast, CTC is obtained with the patient in the supine position.

Question 3. Regarding HRCT and CTC:

(a) Active meningitis and intracranial hypertension are relative contraindications for lumbar puncture.

(b) The sensitivity of CTC is limited by the absence of an active CSF leak.

(c) CTC may be negative when the osseous defect is small, or in low-pressure (low-flow) CSF leaks.

(d) CTC is superior to magnetic resonance (MR) imaging in the detection of meningoencephaloceles.

Question 4. Diagnosis of CSF rhinorrhoea:

(a) Underpneumatised sphenoid sinuses is a common feature in spontaneous CSF leaks involving the sphenoid sinuses.

(b) Osseous-dural defects are commonly associated with arachnoid granulations due to CSF pulsations causing erosion at these sites of anatomical weakness in the base of the skull.

(c) Patients with an enlarged, empty pituitary sella have an increased incidence of spontaneous CSF leak in the sella floor.

(d) The most common site of CSF leak from the anterior cranial fossa involves the frontal sinuses.

Question 5. MR cisternography (MRC) for CSF rhinorrhoea:

(a) The administration of intravenous gadolinium contrast is helpful in the differentiation of CSF column in a fistula from enhancement of mucosal thickening in active sinusitis.

(b) MRC is susceptible to artefacts caused by bone-air interface and is therefore inferior to CT in demonstrating bone defects.

(c) Intrathecal administration of gadolinium contrast is necessary for demonstrating CSF leak.

(d) In cases of inconclusive findings on CT and MRC, the presence of CSF rhinorrhoea can be confirmed by the detection of protein beta-2 transferrin activity in nasal secretions.

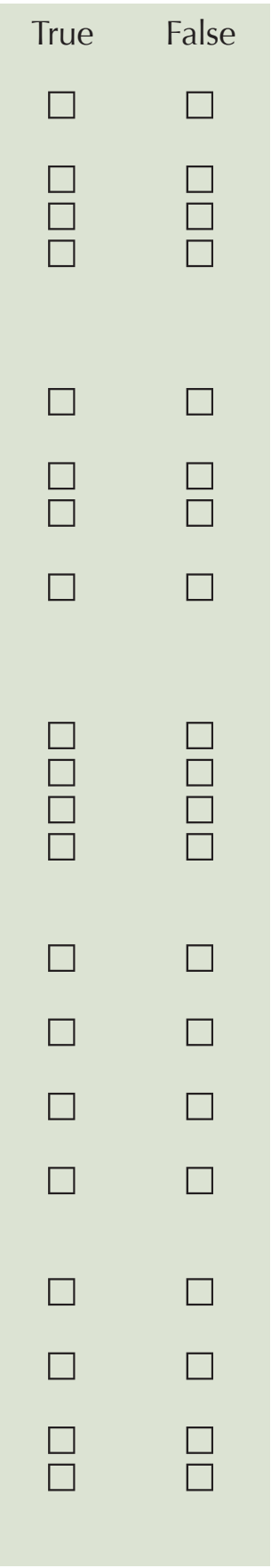

\footnotetext{
Doctor's particulars:

Name in full

MCR number

Specialty:

Email address

(1) Log on at the SMJ website: http://www.sma.org.sg/publications/smjcurrentissue.aspx and select the appropriate set of questions. (2) Provide your name, email address and MCR number. (3) Select your answers and click "Submit".

RESULTS:

(1) Answers will be published in the SMJ May 2013 issue. (2) The MCR numbers of successful candidates will be posted online at the SMJ website by 29 April 2013. (3) Passing mark is $60 \%$. No mark will be deducted for incorrect answers. (4) The SMJ editorial office will submit the list of successful candidates to the Singapore Medical Council. (5) One CME point is awarded for successful candidates.

Deadline for submission: (March 2013 SMJ 3B CME programme): 12 noon, 22 April 2013.
} 